\title{
Neural Sources of Vagus Nerve Stimulation-Induced Slow Cortical Potentials
}

\author{
Borgil Bayasgalan, $\mathrm{MD}, \mathrm{PhD}^{1,2}$; Masao Matsuhashi, $\mathrm{MD}, \mathrm{PhD}^{3}$; \\ Tomoyuki Fumuro, $\mathrm{PhD}^{3,4}$; Naoki Nakano, $\mathrm{MD}, \mathrm{PhD}^{5}$; \\ Masaya Katagiri, MD, $\mathrm{PhD}^{6}$; Akihiro Shimotake, MD, PhD ${ }^{1}$; \\ Takayuki Kikuchi, MD, PhD ${ }^{7}$; Koji lida, MD, $\mathrm{PhD}^{6}$; \\ Takeharu Kunieda, MD, PhD $^{8}$; Amami Kato, MD, PhD ${ }^{5}$; \\ Ryosuke Takahashi, MD, PhD ${ }^{1}$; Akio Ikeda, MD, PhD ${ }^{3}$; Koji Inui, MD, PhD ${ }^{2}$
}

\begin{abstract}
Objectives: This study investigated neuronal sources of slow cortical potentials (SCPs) evoked during vagus nerve stimulation (VNS) in patients with epilepsy who underwent routine electroencephalography (EEG) after implantation of the device.

Materials and Methods: We analyzed routine clinical EEG from 24 patients. There were 5 to 26 trains of VNS during EEG. To extract SCPs from the EEG, a high-frequency filter of $0.2 \mathrm{~Hz}$ was applied. These EEG epochs were averaged and used for source analyses. The averaged waveforms for each patient and their grand average were subjected to multidipole analysis. Patients with at least $50 \%$ seizure frequency reduction were considered responders. Findings from EEG analysis dipole were compared with VNS responses. Results: VNS-induced focal SCPs whose dipoles were estimated to be located in several cortical areas including the medial prefrontal cortex, postcentral gyrus, and insula, with a significantly higher frequency in patients with a good VNS response than in those with a poor response.

Conclusions: This study suggested that some VNS-induced SCPs originating from the so-called vagus afferent network are related to the suppression of epileptic seizures.

Keywords: DC potential, epilepsy, insula, multidipole analysis, prefrontal cortex, VNS

Conflict of Interest: Akio Ikeda and Masao Matsuhashi are affiliated with Department of Epilepsy, Movement Disorders and Physiology, Kyoto University. This department is the Industry-Academia Collaboration Courses, supported by Eisai Co, Ltd., Nihon Kohden Corporation, Otsuka Pharmaceutical Co, Ltd and UCB Japan Co. Ltd. The other authors do not have any conflict of interest.
\end{abstract}

\section{INTRODUCTION}

Epilepsy is a common disorder with multiple etiologies. ${ }^{1}$ Although the best antiepileptic drug treatment is administered, approximately $30 \%$ of patients still have seizures. ${ }^{2}$ For such patients, neurosurgery or neuromodulation techniques, such as vagus nerve stimulation (VNS), are options. Although VNS is an effective and safe treatment option for patients with intractable

Address correspondence to: Borgil Bayasgalan, MD, PhD, Department of Functioning and Disability, Institute for Developmental Research, Aichi Developmental Disability Center, Kamiya-cho 713-8, Kasugai 480-0392, Japan. Email: borgil@inst-hsc.jp

1 Department of Neurology, Graduate School of Medicine, Kyoto University, Kyoto, Japan;

2 Department of Functioning and Disability, Institute for Developmental Research, Aichi Developmental Disability Center, Kasugai, Japan;

3 Department of Epilepsy, Movement Disorders and Physiology, Graduate School of Medicine, Kyoto University, Kyoto, Japan;

${ }^{4}$ Research and Educational Unit of Leaders for Integrated Medical System, Kyoto University, Kyoto, Japan;

${ }^{5}$ Department of Neurosurgery, School of Medicine, Kindai University, Osaka, Japan;

${ }^{6}$ Department of Neurosurgery, School of Medicine, Hiroshima University, Hiroshima, Japan;

7 Department of Neurosurgery, Graduate School of Medicine, Kyoto University, Kyoto, Japan; and

8 Department of Neurosurgery, Graduate School of Medicine, Ehime University, Toon, Japan

For more information on author guidelines, an explanation of our peer review process, and conflict of interest informed consent policies, please see the journal's Guide for Authors.

Source(s) of financial support: This study was funded by the Japan Society for the Promotion of Science (JSPS) KAKENHI Grant Number 20 K16684 to Borgil Bayasgalan. 
epilepsy, $^{3-5}$ the precise mechanisms of its inhibitory action on seizures are largely unknown. ${ }^{6,7}$ Previous neuroimaging studies revealed that some brain areas become active during VNS, and some were more frequently or strongly activated in patients with good responses to VNS than in those with poor responses. These brain areas included the bilateral thalamus and right posterior central sulcus. ${ }^{8}$ This information is useful for understanding the mechanisms of VNS action.

In a previous study, ${ }^{9}$ we examined VNS-induced slow cortical potentials (SCPs) in patients with epilepsy and found an association between the presence of SCPs and seizure reduction, suggesting that alterations on electroencephalography (EEG) because of VNS reflect the efficacy of VNS against seizures. Because SCPs reflect repetitive firing of cortical neurons during VNS, SCPs from specific brain areas may be involved in the suppression of seizures. Therefore, in this study, we investigated the neural sources of SCPS during VNS.

\section{MATERIALS AND METHODS}

\section{Participants}

A total of 24 patients (11 men) with intractable epilepsy aged $28 \pm 17$ (mean \pm standard deviation) years, ranging from 6 to 66 years, at the time of EEG who underwent VNS implantation between November 2010 and August 2014 were examined. This study was approved by the ethics committees of the institutions of all authors. The change in seizure frequency after implantation was assessed as ([number of seizures after VNS per month] - [baseline seizure per month])/(baseline seizure per month). The baseline monthly seizure frequency before VNS was calculated on the last visit before VNS implantation; for patients with more than one visit, the average of up to three monthly visits immediately before the implantation was used. To calculate seizure frequency at the last follow-up visit, the monthly average in the two consecutive months before and in the month of the last follow-up visit was calculated. Depending on the seizure outcome at the time of the last follow-up visit, patients were divided into two groups: responders $(\geq 50 \%$ seizure reduction) and nonresponders ( $<50 \%$ seizure reduction). They all participated in our previous study and their clinical characteristics were described in detail. ${ }^{9}$ At the last follow-up visit (mean 24.1, range 11.7-43.0 months), 12 patients became seizurefree. Patient demographics are briefly described in Supplementary Data Table S1.

\section{VNS Parameters}

VNS was carried out intermittently with VNS ON and VNS OFF times. The VNS ON time was 30 seconds with a ramp-up and rampdown of two seconds, except in one case in which the VNS ON time was 21 seconds. The VNS OFF time was five minutes, three minutes, or 1.8 minutes. The stimulation intensity ranged from 0.75 to 2.00 $\mathrm{mA}$, and stimulation frequency and width were $30 \mathrm{~Hz}$ and $500 \mu \mathrm{s}$, respectively. In two cases, the stimulation intensity was not available (Table S1).

\section{EEG Recordings}

We analyzed 30-minute routine EEG from patients who were followed up after VNS implantation. EEG was recorded using EEG1100 or EEG2100 (Nihon Kohden, Tokyo, Japan). Depending on the examining institution, 19 or 21 scalp electrodes and two earlobe electrodes were placed according to the International 10-20 system. EEG recording was performed after a mean of 12.2 (range 4.2-36.7) months from VNS implantation. The sampling rate was 200 or $500 \mathrm{~Hz}$, and the time constant was 2 or 10 seconds.

\section{SCP Analysis}

The analysis window was a period from two seconds before to 34 seconds after the onset of each VNS. The two-second period before VNS was used as the baseline. Epochs with the following artifacts were excluded from analyses ${ }^{9}$ :

- initial 10 minutes of recording if baseline shifts $>150 \mu \mathrm{V}$ were observed during the initial 60 seconds of the recording;

- recording reset because of pausing of recording or montage change in certain EEG systems;

- large movement artifacts $>400 \mu \mathrm{V}$; and

- temporally overlapping eye movement potentials observable on raw EEG.

To focus on slow potentials, a low-pass filter of $0.2 \mathrm{~Hz}$ was applied to EEG.

\section{Dipole analysis}

EEG epochs of VNS were averaged for each participant and the averaged waveforms were used for dipole source analysis using brain electrical source analysis (BESA Research 5.3; BESA GmbH, Gräefelfing, Germany), as described previously. ${ }^{10}$ In brief, model adequacy was assessed by examining: 1) the percentage of variance and 2) the $F$ ratio (ratio of reduced $x^{2}$ values before and after adding a new source). ${ }^{11}$ New dipoles with an $F$ ratio of $p$ values $<0.05$ were considered significant. Because activities near the eye and in the occipital region were frequent but considered to be nonspecific to VNS, they were excluded before the dipole analysis using a principal component analysis, if present. We continued to add dipoles until the additional dipole did not significantly improve the fit.

We analyzed waveforms for each participant and grandaveraged waveforms across all participants. To average EEG recordings with slightly different electrode configurations, spatial interpolation to 81 standard electrodes re-referenced to the average reference was used.

\section{RESULTS}

After the application of our criteria, 135 VNS epochs were selected from 24 participants $(5.6 \pm 3.7$ epochs for each participant). The grand-averaged waveforms across participants are shown in Figure 1a. The most frequent dipolar pattern field distribution across all time periods is presented in the isocontour map in Figure $1 \mathrm{bi}$. The main generator to explain the field was estimated to be located in the lateral part of the right central sulcus corresponding to the primary somatosensory and motor cortex (dipole 1). The theoretical electric field caused by the model is shown in the right panel in Figure 1bi. After subtraction of waveforms that were explained by the first dipole, a dipolar pattern distribution remained in the left parietal region. The best second dipole to explain the residual waveforms was estimated to be located in the left central gyrus (dipole 2). The second dipole covered a wide range of recorded data and by adding the second dipole, goodness of fit was significantly improved, for example, from $62.7 \%$ to $79.5 \%$ at 16,000 ms from the VNS onset (Fig. 1 bii, $\mathrm{F}=1.68, p=0.014$ ). Clear dipolar pattern fields that were unable to be explained by the 
a

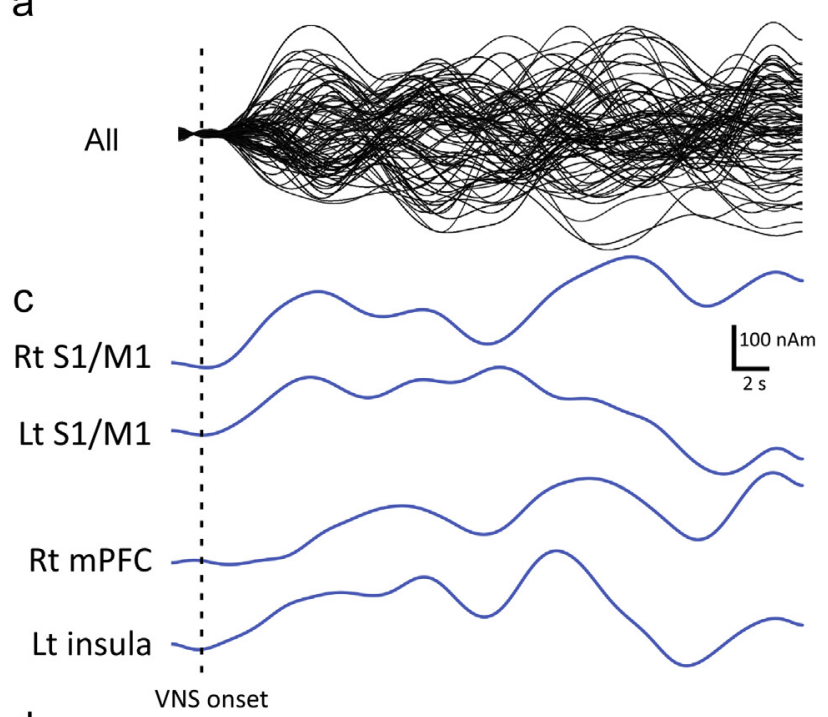

b

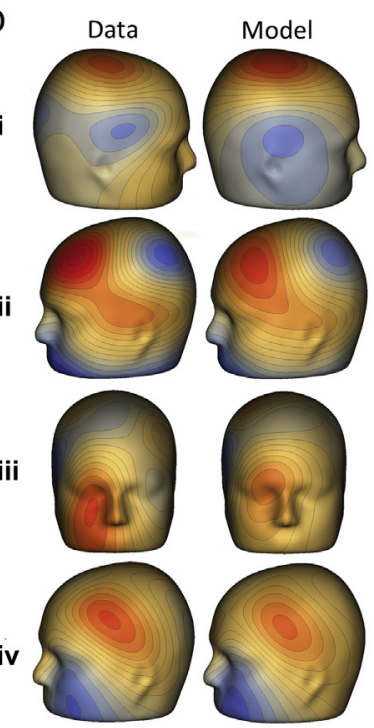

d
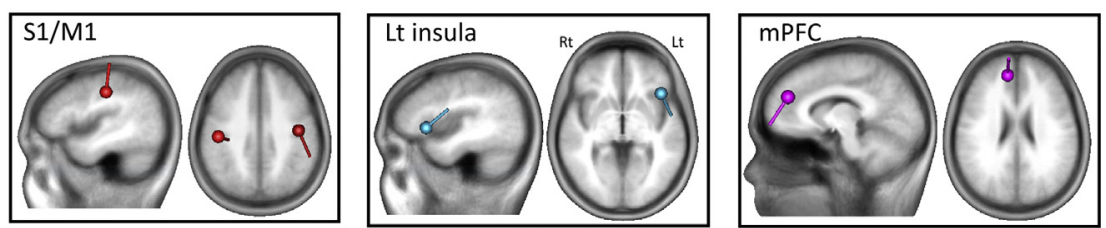

Figure 1. Dipole analyses. a. Grand-averaged waveforms across 24 participants. b. Scalp electric field distributions of recorded data (left) and models (right). c. Source strength waveforms for each dipole. d. Location of estimated dipoles superimposed on sagittal and transverse slices of a standard brain. M1, primary motor cortex; mPFC, medial prefrontal cortex; Lt, left; Rt, right; S1, primary somatosensory cortex. [Color figure can be viewed at www.neuromodulationjournal.org]

two-dipole model remained (Figs. 1 biii and 1 biv). After similar procedures, dipole 3 and dipole 4 were estimated in the right medial prefrontal gyrus $(\mathrm{F}=2.10, p=0.0013)$ and left insula $(\mathrm{F}=$ $\left.1.27, p=8.5 \times 10^{-5}\right)$. The time course of each cortical source and the dipole location are shown in Figure $1 c, d$. The $x, y$, and $z$ coordinates of each dipole are listed in Table 1.

Similar analyses were performed for each patient. The plots of all estimated dipoles in responders and nonresponders are shown in Figure 2. Significant dipoles were estimated in 9 of 12 responders and 3 of 12 nonresponders. Dipoles were more frequent in responders ( $p=0.039$, Fisher exact test). The most frequent dipole was that in the right central sulcus (four in responders and one in nonresponders). The remaining dipoles were localized in the left central sulcus, medial prefrontal cortex, left insula, and dorsal prefrontal cortex. Sex, VNS parameters (stimulation interval, pulse intensity, and pulse width), interval from implantation to EEG, and age did not significantly affect VNS effectiveness or the presence of dipoles (Table 2). Although statistical analyses were not performed, VNS effectiveness was equal for all types of epilepsy (Table 3 ).

\section{DISCUSSION}

This study investigated neural sources responsible for VNSinduced SCPs using multiple dipole analysis. Dipoles were estimated to be located in the postcentral gyrus, insula, dorsolateral prefrontal cortex, and medial prefrontal cortex and were significantly more frequent in the responder group. In particular, insular activation was exclusive to the responder group. Therefore, we considered at least some of the VNS-induced SCPs to reflect activity related to the inhibition of seizures.

There are several known SCPs in humans, including sustained brain responses to repeated sensory stimuli, contingent negative variations (CNV), and readiness potentials. ${ }^{12}$ Unlike readiness potentials and CNV that are induced by internal cues, such as expectancy, sustained cortical responses to repeated sensory stimuli are elicited by external inputs. The sustained potential following sensory stimuli can be recorded during sleep or without the participants' attention and is therefore considered an exogenous sensory response. ${ }^{13}$ Because the SCPs in this study were

Table 1. Dipole Location.

\begin{tabular}{|c|c|c|c|c|}
\hline Source & Tal $(x)$ & Tal (y) & Tal (z) & Region \\
\hline Dipole 1 & 44 & -21 & 37 & Postcentral gyrus (S1)/precentral gyrus (M1) \\
\hline Dipole 2 & -41 & -14 & 36 & Postcentral gyrus (S1)/precentral gyrus (M1) \\
\hline Dipole 3 & 10 & 44 & 25 & Medial prefrontal cortex \\
\hline Dipole 4 & -41 & 20 & -1 & Anterior insula \\
\hline
\end{tabular}



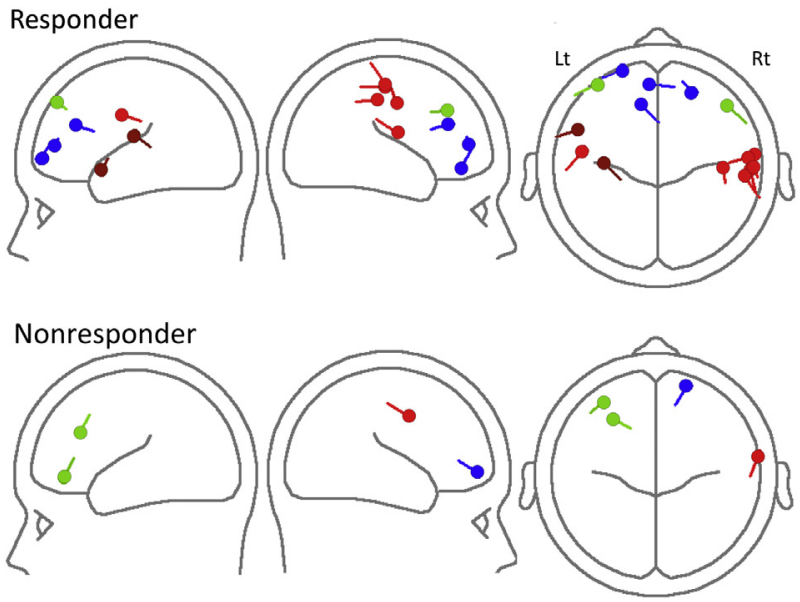

Figure 2. Location of all estimated dipoles. Dipoles in red, blue, green, and brown indicate dipoles in the postcentral gyrus, medial prefrontal cortex, lateral prefrontal cortex, and insula, respectively. Lt, left; Rt, right. [Color figure can be viewed at www.neuromodulationjournal.org]

elicited by VNS without the participants' awareness, they may have an electrophysiological nature similar to that of sustained SCPs evoked by sensory stimuli. The VNS activating the lateral part of the postcentral gyrus and insula is consistent with previous studies reporting that the sensory cortex of each modality is the origin for sustained cortical responses to auditory, ${ }^{14}$ somatosensory, ${ }^{15}$ and visual $^{16}$ stimuli. In addition to these well-known measures in the research field of event-related potentials, focal SCPs accompanying epileptic seizures ${ }^{17,18}$ are important for understanding the neural mechanisms of SCPs. Several cellular mechanisms are involved in epilepsy-related SCPs, including hyperpolarization of glial cells and increased extracellular $\mathrm{K}^{+}$concentration. ${ }^{19} \mathrm{~A}$ similar role of glial cells also is known in stimulus-evoked $\mathrm{SCPs}^{20}$ and may therefore be related to the VNS-induced SCPs. We considered the SCPs in this study to reflect prolonged focal activation in several cortical areas.

\section{Physiological Significance of Each Cortical Source}

The exact inhibitory mechanisms of VNS that regulate epilepsy are not known. One approach is to examine brain regions that respond to VNS. Ideally, this may reveal brain regions connected to the clinical effectiveness of VNS. Previous studies using noninvasive imaging techniques, such as functional magnetic resonance imaging (fMRI), positron emission tomography (PET), and singlephoton emission computed tomography (SPECT), demonstrated significant activation in some brain regions including the thalamus, limbic system, insula, and prefrontal cortex. ${ }^{21-28}$ Although this study was unable to provide a definite conclusion, similar to previous studies, there were several regions activated by VNS more frequently in responders that may reflect the antiepileptic action of VNS.

\section{Primary Somatosensory Cortex}

The most frequent dipole in the analyses of each participant was in and around the central sulcus in the right hemisphere. As shown in Table 1, its location corresponded to the visceral region of the primary somatosensory cortex (S1), one of the regions activated by the stimulation of digestive organs., ${ }^{8-29-32}$ Similar regions were reported to be activated by VNS. ${ }^{8,25}$ A PET study measured brain blood flow just after the start of VNS and three months later and found that the right postcentral gyrus was activated in both scans. ${ }^{8}$ This activation may come from the somatosensory events of the cervical region during VNS, but in this study and in a previous fMRI study $^{25}$ activation was observed bilaterally. Therefore, we considered S1 activation to be caused by the stimulation of vagus afferents. It also may reflect the effective activation of the thalamocortical pathway during VNS. Of note, Mithani et $\mathrm{al}^{33}$ recorded somatosensory evoked fields (SEFs) following median nerve stimulation in patients with epilepsy and found that responders to VNS had more widespread SEF localization and greater functional connectivity within the limbic and sensorimotor networks than nonresponders. Therefore, this study may reflect more widespread projections to S1 from the thalamus in responders.

Table 2. Patient Demographics and VNS Effectiveness.

\begin{tabular}{|c|c|c|c|c|c|c|}
\hline Variables & \multicolumn{2}{|c|}{ VNS effectiveness } & $p$ Value & \multicolumn{2}{|c|}{ Dipoles estimated } & $p$ Value \\
\hline Age at EEG & $33.2 \pm 19.2$ & $24.4 \pm 13.7$ & $0.68^{*}$ & $29.8 \pm 16.4$ & $27.9 \pm 17.2$ & $1.00^{*}$ \\
\hline Male & 5 & 6 & \multirow[t]{2}{*}{$1.00^{\dagger}$} & 6 & 5 & \multirow[t]{2}{*}{$1.00^{\dagger}$} \\
\hline Female & 7 & 6 & & 6 & 7 & \\
\hline \multicolumn{7}{|l|}{ VNS interval (min) } \\
\hline \multicolumn{7}{|l|}{ Pulse intensity } \\
\hline$>1.5 \mathrm{~mA}$ & 5 & 2 & \multirow[t]{2}{*}{$0.38^{+}$} & 5 & 2 & \multirow[t]{2}{*}{$0.37^{\dagger}$} \\
\hline$\leq 1.5 \mathrm{~mA}$ & 7 & 8 & & 6 & 9 & \\
\hline Interval from implantation to EEG (months) & $14.1 \pm 9.7$ & $10.3 \pm 5.8$ & $1.00^{*}$ & $13.9 \pm 10.0$ & $10.5 \pm 6.2$ & $0.32^{*}$ \\
\hline Interval from implantation to last follow-up (mo) & $28.3 \pm 8.0$ & $20.3 \pm 6.4$ & $0.04^{*}$ & & & \\
\hline \multicolumn{7}{|l|}{ Dipole estimation } \\
\hline
\end{tabular}


Table 3. Correlation Between Response to VNS and Estimated Dipoles and Diagnosis and Seizure Types.

\begin{tabular}{lllll}
$\begin{array}{l}\text { Diagnosis/seizure } \\
\text { type }\end{array}$ & \multicolumn{2}{l}{ VNS responsiveness } & \multicolumn{2}{l}{ Dipoles estimated } \\
& Resp & Nonresp & Present & Absent \\
Diagnosis & & & & \\
FLE & 2 & 2 & 2 & 2 \\
SGE & 1 & 2 & 2 & 1 \\
SPE & 3 & 4 & 4 & 3 \\
LGS & 4 & 2 & 2 & 4 \\
TLE & 2 & 1 & 2 & 1 \\
OLE & 0 & 1 & 0 & 1 \\
Seizure type* & & & & \\
CPS & 5 & 9 & 6 & 8 \\
GTCS and sGTCS & 6 & 3 & 6 & 3 \\
SPS & 2 & 4 & 3 & 3 \\
AAS & 3 & 0 & 1 & 2 \\
TS & 3 & 1 & 1 & 3 \\
MS & 0 & 1 & 1 & 0 \\
Spasms & 1 & 2 & 2 & 1 \\
HD & 2 & 0 & 0 & 2 \\
AS & 1 & 0 & 0 & 1
\end{tabular}

AAS, atypical absence seizures; AS, atonic seizures; Bil, bilateral; CPS, complex partial seizures; FLE, frontal lobe epilepsy; GTCS, generalized tonic-clonic seizures; HD, head drop; L, left; LGS, Lennox-Gastaut syndrome; MS, myoclonic seizures; N/A, not available; Nonresp, nonresponders; OLE, occipital lobe epilepsy; R, right; Resp, responders; SGE, symptomatic generalized epilepsy; sGTCS, secondarily generalized tonic-clonic seizures; spasms, spasm seizures; SPE, symptomatic partial epilepsy; SPS, simple partial seizures; TLE, temporal lobe epilepsy; TS, tonic seizures.

*Because of multiple seizure types for a single patient, the total number exceeds 24.

\section{Medial Prefrontal Cortex}

The medial prefrontal cortex is one of the cortical regions that receive visceral inputs and participate in controlling autonomic functions. ${ }^{34,35}$ Therefore, its activation in this study is reasonable and consistent with previous studies measuring cerebral blood flow during $\mathrm{VNS}^{8,21}$ and during anal canal stimulation. ${ }^{31}$ Ibrahim et al ${ }^{36}$ examined thalamocortical connectivity using resting-state fMRI before VNS implantation in children with intractable epilepsy and found that patients with a good response to VNS exhibited significantly stronger connectivity of the thalamus with the bilateral insula and anterior cingulate/ventromedial prefrontal cortex than those with a poor response, suggesting that this region is involved in the suppressive effects of VNS on epileptic seizures.

Insula

The insula is a part of the central autonomic network ${ }^{37}$ and receives visceral information through the nucleus of the solitary tract and parabrachial nucleus. In addition to animal studies, noninvasive neuroimaging techniques revealed its important position in the brain organization related to visceral afferent inputs in humans. ${ }^{34}$ In imaging studies, activation of the insula was observed following stimulation of the digestive tract ${ }^{29-31}$ and following VNS. 8,23,25,27 The insula is part of the orbital network of the prefrontal cortex subserving the visceral control. ${ }^{38}$ Although several imaging studies reported bilateral activation of the insula, ${ }^{8,25}$ insular dipoles were predominant for the left side based on both the averaged and individual data. In addition, insular activation was not observed in the nonresponder group, suggesting that left-sided VNS activates the ipsilateral insula and may be an indication of good responsiveness to VNS.

Of note, a large sample study reported that VNS responders possessed more robust left-lateralized white matter microstructures within a specific network. ${ }^{39}$ Such a network related to VNS treatment effects, the vagus afferent network, ${ }^{40}$ includes brainstem structures such as the nucleus of the solitary tract in addition to subcortical or cortical structures such as the insula, amygdala, thalamus, prefrontal cortex, anterior cingulate cortex, and S1. Thus, the dipoles in this study are consistent with the vagus afferent network.

\section{Clinical Significance}

In addition to epilepsy, the efficacy of VNS has been investigated for a variety of diseases such as depression and headache. ${ }^{41,42}$ Moreover, because of its cholinergic anti-inflammatory effects, ${ }^{43}$ VNS has been applied to inflammatory diseases including sepsis, chronic pain, and bowel diseases. ${ }^{44,45}$ Some effects of VNS may originate from the anti-inflammatory properties of vagus nerve efferents, but VNS should exert its effects through vagus nerve afferents in many cases. Therefore, VNS-induced brain activation in this study may be related to diseases other than epilepsy. For example, the medial prefrontal cortex plays a role in autonomic control, stress reactivity, ${ }^{35}$ and inflammation-induced mood change ${ }^{46}$ in addition to controlling serotonergic function. ${ }^{47}$ The former is related to depression or stress-related psychiatric disorders and the latter to seizures. ${ }^{48}$ Furthermore, the medial prefrontal cortex regulates the baroreflex such that it participates in a central sympathoinhibitory pathway. ${ }^{49}$ The baroreflex, in turn, induces a trophotropic state ${ }^{50}$ including inhibition of sham rage, ${ }^{51}$ inactivation of pyramidal tract neurons, ${ }^{52}$ and suppression of seizures. ${ }^{53}$ Thus, it is of interest to clarify the roles of the medial prefrontal cortex-baroreflex arc connection in the VNS action against different diseases because afferents of the baroreceptor run along the cervical vagus nerve and must be activated during VNS. Exploring the neural generators of VNS-induced SCPs is useful not only for the evaluation of treatment responsiveness in individual patients but also for understanding the pathophysiology of related diseases.

\section{Limitations}

There are some limitations in this study. First is the method for source localization. The current method is unique in that we focused on brain activity in a specific frequency band, namely, specific brain activity related to VNS. However, there are other methods for localizing brain activation. The dipole estimation used in this study is weak in that EEG cannot detect activity from the socalled closed field, such as the thalamus, and that resolving inverse problems has a risk of calculation errors. In addition, this study did not use high-density EEG, which can facilitate dipole localization of scalp potentials. However, the current method has advantages in that it provides information from specific frequency bands in a routine EEG examination. This was important in this study because we focused on SCPs. In contrast, FMRI, SPECT, and PET can detect activation in deep brain areas and do not require resolving of inverse problems. Second, the sample was heterogeneous. Although we noted no effects of age, sex, or VNS parameters on the VNS effectiveness, the sample size was insufficient to draw a 
definite conclusion. Third, because this was a multi-institutional study, procedures were slightly different among patients. For example, the minimum follow-up period was not consistent among institutes, which may have affected the results because it is known that responses to VNS increase over time.

\section{Conclusion}

To localize the VNS-induced SCPs, we retrospectively analyzed EEG from 24 patients with epilepsy who were undergoing VNS treatment. We performed dipole analyses to localize neural origins of VNS-induced SCPs. Dipoles were estimated to be located in the so-called vagus afferent network and were significantly more frequent in VNS responders, supporting the view that such a network is related to VNS treatment effectiveness.

\section{Authorship Statements}

Borgil Bayasgalan and Koji Inui prepared the manuscript draft. Masao Matsuhashi, Tomoyuki Fumuro, Akio Ikeda, and Akihiro Shimotake participated in the analysis of data. Amami Kato, Naoki Nakano, Koji Inui, Masaya Katagiri, Takeharu Kunieda, Takayuki Kikuchi, and Akio Ikeda provided data. Akio Ikeda and Ryosuke Takahashi supervised the research. The final manuscript was critically revised and approved by all authors.

\section{How to Cite This Article}

Bayasgalan B., Matsuhashi M., Fumuro T., Nakano N., Katagiri M., Shimotake A., Kikuchi T., lida K., Kunieda T., Kato A., Takahashi R., Ikeda A., Inui K. 2022. Neural Sources of Vagus Nerve Stimulation-Induced Slow Cortical Potentials.

Neuromodulation 2022; 25: 407-413.

\section{SUPPLEMENTARY DATA}

To access the supplementary material accompanying this article, visit the online version of Neuromodulation: Technology at the Neural Interface at www.neuromodulationjournal.org and at https://doi.org/10.1016/j.neurom.2022.01.009.

\section{REFERENCES}

1. Shorvon SD. The etiologic classification of epilepsy. Epilepsia. 2011;52:1052-1057.

2. Chen Z, Brodie MJ, Liew D, Kwan P. Treatment outcomes in patients with newly diagnosed epilepsy treated with established and new antiepileptic drugs: a 30year longitudinal cohort study. JAMA Neurol. 2018;75:279-286.

3. Ben-Menachem E, Mañon-Espaillat R, Ristanovic R, et al. Vagus nerve stimulation for treatment of partial seizures: 1. A controlled study of effect on seizures. First International Vagus Nerve Stimulation Study Group. Epilepsia. 1994;35:616-626.

4. Majoie HJM, Berfelo MW, Aldenkamp AP, Renier WO, Kessels AGH. Vagus nerve stimulation in patients with catastrophic childhood epilepsy, a 2-year follow-up study. Seizure. 2005;14:10-18.

5. Shahwan A, Bailey C, Maxiner W, Harvey AS. Vagus nerve stimulation for refractory epilepsy in children: more to VNS than seizure frequency reduction. Epilepsia. 2009;50:1220-1228.

6. Loddenkemper T, Alexopoulos AV. Vagus nerve stimulation: human studies. In: Luders HO, ed. Textbook of Epilepsy Surgery. Informa Healthcare; 2008:1188-1200.

7. Fanselow EE. Central mechanisms of cranial nerve stimulation for epilepsy. Surg Neurol Int. 2012;3:S247-S254.

8. Henry TR, Bakay RAE, Pennell PB, Epstein CM, Votaw JR. Brain blood-flow alterations induced by therapeutic vagus nerve stimulation in partial epilepsy: II.
Prolonged effects at high and low levels of stimulation. Epilepsia. 2004:45: 1064-1070.

9. Bayasgalan B, Matsuhashi M, Fumuro T, et al. We could predict good responders to vagus nerve stimulation: a surrogate marker by slow cortical potential shift. Clin Neurophysiol. 2017;128:1583-1589.

10. Inui $K$, Wang $X$, Tamura $Y$, Kaneoke $Y$, Kakigi R. Serial processing in the human somatosensory system. Cereb Cortex. 2004;14:851-857.

11. Supek S, Aine CJ. Simulation studies of multiple dipole neuromagnetic source localization: model order and limits of source resolution. IEEE Trans Biomed Eng. 1993:40:529-540.

12. Birbaumer N, Elbert T, Canavan AG, Rockstroh B. Slow potentials of the cerebral cortex and behavior. Physiol Rev. 1990;70:1-41.

13. Picton TW, Woods DL, Proulx GB. Human auditory sustained potentials. I. The nature of the response. Electroencephalogr Clin Neurophysiol. 1978:45:186197.

14. Hari R, Aittoniemi K, Järvinen ML, Katila T, Varpula T. Auditory evoked transient and sustained magnetic fields of the human brain. Localization of neural generators. Exp Brain Res. 1980;40:237-240.

15. Hagiwara K, Ogata K, Hironaga N, Tobimatsu S. Secondary somatosensory area is involved in vibrotactile temporal-structure processing: MEG analysis of slow cortical potential shifts in humans. Somatosens Mot Res. 2020;37:222-232.

16. Järvilehto T, Hari R, Sams M. Effect of stimulus repetition on negative sustained potentials elicited by auditory and visual stimuli in the human EEG. Biol Psychol. 1978;7:1-12.

17. Ikeda A, Terada K, Mikuni N, et al. Subdural recording of ictal DC shifts in neocortical seizures in humans. Epilepsia. 1996:37:662-674.

18. Ikeda A, Taki W, Kunieda T, et al. Focal ictal direct current shifts in human epilepsy as studied by subdural and scalp recording. Brain. 1999;122:827-838.

19. Somjen GG. Electrogenesis of sustained potentials. Prog Neurobiol. 1973;1:201-237.

20. Castellucci VF, Goldring S. Contribution to steady potential shifts of slow depolarization in cells presumed to be glia. Electroencephalogr Clin Neurophysiol. 1970;28:109-118.

21. Garnett ES, Nahmias C, Scheffel A, Firnau G, Upton AR. Regional cerebral blood flow in man manipulated by direct vagal stimulation. Pacing Clin Electrophysiol. 1992;15:1579-1580.

22. Ko D, Heck C, Grafton S, et al. Vagus nerve stimulation activates central nervous system structures in epileptic patients during PET H2(15)O blood flow imaging. Neurosurgery. 1996;39:426-430.

23. Henry TR, Bakay RAE, Votaw JR, et al. Brain blood flow alterations induced by therapeutic vagus nerve stimulation in partial epilepsy: I. Acute effects at high and low levels of stimulation. Epilepsia. 1998;39:983-990.

24. Bohning DE, Lomarev MP, Denslow S, Nahas Z, Shastri A, George MS. Feasibility of vagus nerve stimulation-synchronized blood oxygenation level-dependent functional MRI. Invest Radiol. 2001;36:470-479.

25. Narayanan JT, Watts R, Haddad N, Labar DR, Li PM, Filippi CG. Cerebral activation during vagus nerve stimulation: a functional MR study. Epilepsia. 2002;43:15091514.

26. Sucholeiki R, Alsaadi TM, Morris GL 3rd, Ulmer JL, Biswal B, Mueller WM. fMRI in patients implanted with a vagal nerve stimulator. Seizure. 2002;11:157-162.

27. Liu WC, Mosier K, Kalnin AJ, Marks D. BOLD fMRI activation induced by vagus nerve stimulation in seizure patients. J Neurol Neurosurg Psychiatry. 2003;74:811-813.

28. Vonck K, Boon P. The mechanism of action of vagus nerve stimulation therapy. Eur Neurol Rev. 2008:3:97-100.

29. Aziz Q, Andersson JL, Valind S, et al. Identification of human brain loci processing esophageal sensation using positron emission tomography. Gastroenterology. 1997;113:50-59.

30. Hecht M, Kober H, Claus D, Hilz M, Vieth J, Neundörfer B. The electrical and magnetical cerebral responses evoked by electrical stimulation of the esophagus and the location of their cerebral sources. Clin Neurophysiol. 1999:110:1435-1444.

31. Hobday DI, Aziz Q, Thacker N, Hollander I, Jackson A, Thompson DG. A study of the cortical processing of ano-rectal sensation using functional MRI. Brain. 2001:124:361-368.

32. Aziz Q, Thompson DG, Ng VW, et al. Cortical processing of human somatic and visceral sensation. J Neurosci. 2000;20:2657-2663.

33. Mithani $\mathrm{K}$, Wong SM, Mikhail M, et al. Somatosensory evoked fields predict response to vagus nerve stimulation. Neuroimage Clin. 2020;26:102205.

34. Cechetto DF. Cortical control of the autonomic nervous system. Exp Physiol. 2014;99:326-331.

35. McKlveen JM, Myers B, Herman JP. The medial prefrontal cortex: coordinator of autonomic, neuroendocrine and behavioural responses to stress. J Neuroendocrinol. 2015;27:446-456.

36. Ibrahim GM, Sharma P, Hyslop A, et al. Presurgical thalamocortical connectivity is associated with response to vagus nerve stimulation in children with intractable epilepsy. Neurolmage Clin. 2017;16:634-642.

37. Benarroch EE. The central autonomic network: functional organization, dysfunction, and perspective. Mayo Clin Proc. 1993;68:988-1001.

38. Öngür D, Price JL. The organization of networks within the orbital and medial prefrontal cortex of rats, monkeys and humans. Cereb Cortex. 2000;10:206-219.

39. Mithani $K$, Mikhail M, Morgan BR, et al. Connectomic profiling identifies responders to vagus nerve stimulation. Ann Neurol. 2019;86:743-753.

40. Hachem LD, Wong SM, Ibrahim GM. The vagus afferent network: emerging role in translational connectomics. Neurosurg Focus. 2018:45:E2. 
41. Bottomley JM, LeReun C, Diamantopoulos A, Mitchell S, Gaynes BN. Vagus nerve stimulation (VNS) therapy in patients with treatment resistant depression: a systematic review and meta-analysis. Compr Psychiatry. 2020;98:152156.

42. Lendvai IS, Maier A, Scheele D, Hurlemann R, Kinfe TM. Spotlight on cervical vagus nerve stimulation for the treatment of primary headache disorders: a review. J Pain Res. 2018;11:1613-1625.

43. Bonaz B, Picq C, Sinniger V, Mayol JF, Clarençon D. Vagus nerve stimulation: from epilepsy to the cholinergic anti-inflammatory pathway. Neurogastroenterol Motil. 2013;25:208-221.

44. Johnson $\mathrm{RL}$, Wilson $\mathrm{CG}$. A review of vagus nerve stimulation as a therapeutic intervention. J Inflamm Res. 2018;11:203-213.

45. Bonaz B. Is-there a place for vagus nerve stimulation in inflammatory bowel diseases? Bioelectron Med. 2018:4:4.

46. Harrison NA, Brydon L, Walker C, Gray MA, Steptoe A, Critchley HD. Inflammation causes mood changes through alterations in subgenual cingulate activity and mesolimbic connectivity. Biol Psychiatry. 2009;66:407-414.

47. Martín-Ruiz R, Puig MV, Celada P, et al. Control of serotonergic function in medial prefrontal cortex by serotonin-2A receptors through a glutamate-dependent mechanism. J Neurosci. 2001;21:9856-9866.

48. Bagdy G, Kecskemeti V, Riba P, Jakus R. Serotonin and epilepsy. J Neurochem. 2007; 100:857-873.

49. Resstel LB, Corrêa FM. Involvement of the medial prefrontal cortex in central cardiovascular modulation in the rat. Auton Neurosci. 2006;126-127:130-138.

50. Inui K, Nomura J, Murase S, Nosaka S. Facilitation of the arterial baroreflex by the preoptic area in anaesthetized rats. J Physiol. 1995;488:521-531.

51. Zanchetti A. Reflex and brain stem inhibition of sham rage behaviour. Prog Brain Res. 1968;22:195-205.

52. Coleridge HM, Coleridge JC, Rosenthal F. Prolonged inactivation of cortical pyramidal tract neurones in cats by distension of the carotid sinus. J Physiol. 1976;256:635-649.

53. Gellhorn E, Yesinick L, Kessler M, Hailman H. Carotid sinus reflexes and convulsions. Am J Physiol. 1942;137:396-403.

\section{COMMENTS}

VNS has been found to be effective for treating patients with epilepsy, but the mechanism(s) of the inhibitory action of VNS on seizures has not been clearly established. This article describes the findings from an analysis of VNS and slow cortical potentials (SCPS) obtained from EEG data collected at one time-point after VNS implantation in an age-diverse group of 24 patients with various epilepsy subtypes. The objective of the study is to use a dipole approach for cortical localization of SCPs and to correlate these dipole locations with response and nonresponse to VNS. Although the dipole approach used by the authors is only one possible method for localization, and the study has a number of methodological limitations, it is hoped that this study will spur further research on identifying valid mechanisms of action of VNS

Robert Howland, MD

Pittsburgh, PA, USA

The current work provides additional insights into neural networks engaged by VNS. A series of investigations encompassing different modalities converge upon similar brain regions that are functionally connected and engaged by VNS. This so-called "vagus afferent network" provides a framework to study VNS efficacy and responsiveness to treatment. The authors show that induced focal slow potentials originate in the regions of the vagus afferent network, further expanding our understanding of this network and the mechanisms of action of VNS.

George M. Ibrahim, MD, PhD Toronto, ON, Canada

This is an interesting article investigating neural sources of SCPS during VNS. These findings could improve our understanding of the mechanism of action and affected brain regions by VNS.

Ann Mertens, MD Ghent, Belgium 